\title{
PRIMARY ASYMPTOMATIC CALGIFICATION OF ARTICULAR CARTILAGE
}

\author{
Report of a Case \\ D. A. W. Edwards and S. Davis, London, ENGland \\ From the Department of Clinical Research, L'nicersity College Hospital .Medical School,* \\ and the Department of Radiology', Eniversity College Hospital
}

A surveyor, aged forty-five, complained of pain, tenderness, and swelling in his right knee, of two weeks duration. At first mild, the pain increased to moderate severity and was aggravated by movement. There was no history of injury, locking, or limitation of movement. He had had intermittent brief attacks of mild pain and swelling of both knees, left ankle, and right shoulder during the previous three years.

On examination the right knee was warmer than the left; there was periarticular swelling

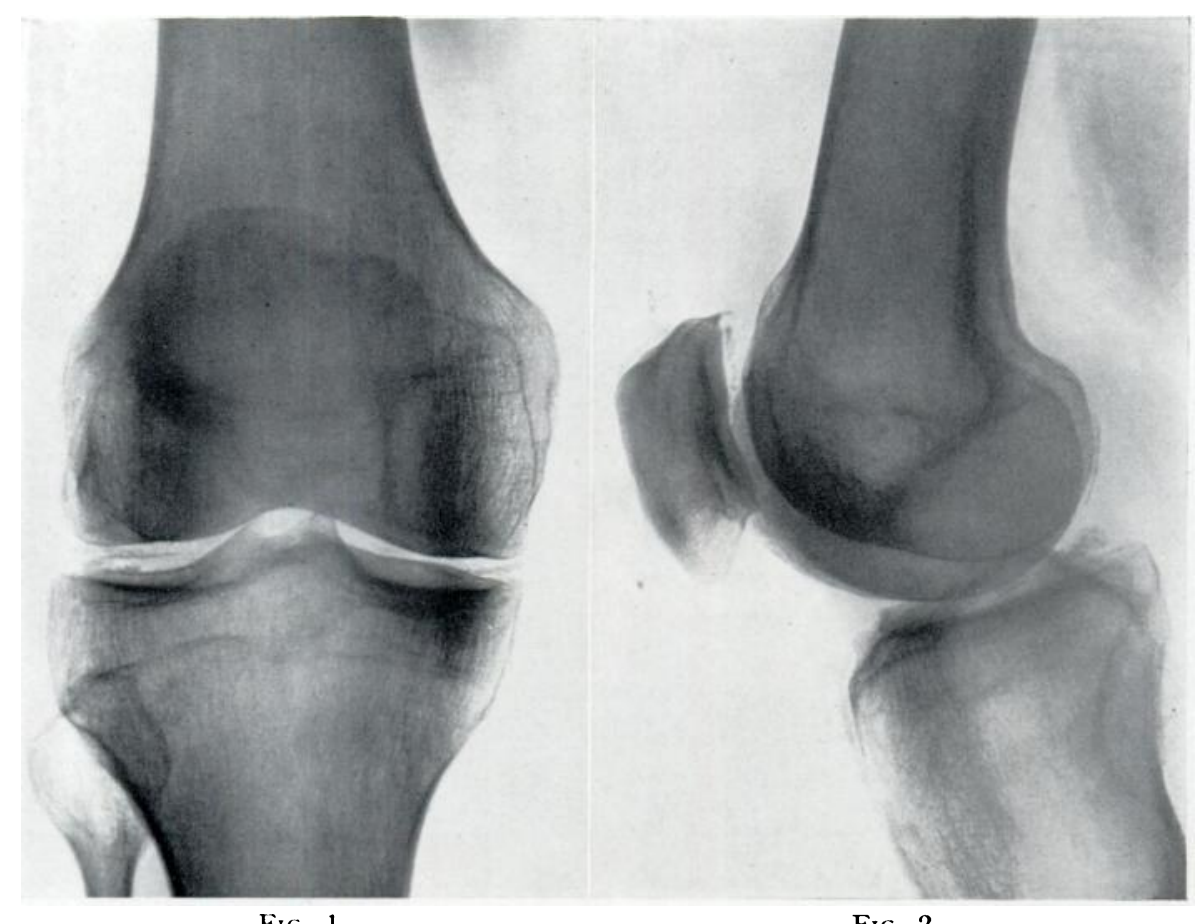

Right knee. Figure 1--Calcification of a granular type is seen in both semilunar cartilages.

Figure 2-lavers of calcium can be seen on the deep surface of the patella, and parallel to the articular condyle of the femur. The suprapatellar tendon is also calcified.

and an effusion of fluid in the joint. There was tenderness over the medial cartilage adjacent to the medial ligament. The range of movement was full. The other joints showed no abnormality and there were no signs of disease elsewhere. The erythrocyte sedimentation rate was 1:- millimetres at one hour (Westergren).

Progress-The symptoms and signs subsided within four weeks without treatment other than rest in bed. Four months later he had further slight pain in the right knee for four days, but without swelling.

\footnotetext{
* Work undertaken on behalf of the Medical Research Council.
} 
Radiographic examination. Knees-There were osteoarthritic changes in both knees, but the most striking changes were in the articular and semilunar cartilages. Throughout all four semilunar cartilages there was a flocculent granular calcification, densest towards the surface and more marked in the lateral cartilages (Figs. 1 and 2 ). In each lateral projection there was a layer of calcification about a millimetre thick lying parallel to the posterior surface of the femoral condyles and separated from the cortical bone by a translucent zone. This layer bore no relationship to the calcified cartilages. Similar layers were seen parallel to the articular surfaces of the patellae. Both suprapatellar ligaments were calcified and there was some calcification within the articular cartilage of the right superior tibio-fibular joint.

It was evident from its shape and position that the calcium deposit lay in the more superficial layers of the articular cartilage, with short frond-like extensions into the deeper layers. At no point did the calcification reach the bone cortex. The appearance was compatible with either calcification of the superficial layers of articular cartilage cells, or an incrustation of calcium-containing material upon a deeply and extensively fissured articular surface. Shoulders-In the left shoulder a calcified layer a millimetre thick overlay the greater part of the projection of the head of the humerus (Fig. 3). The glenoid cavity contained a similar opaque layer of even thickness, in spite of the heaping

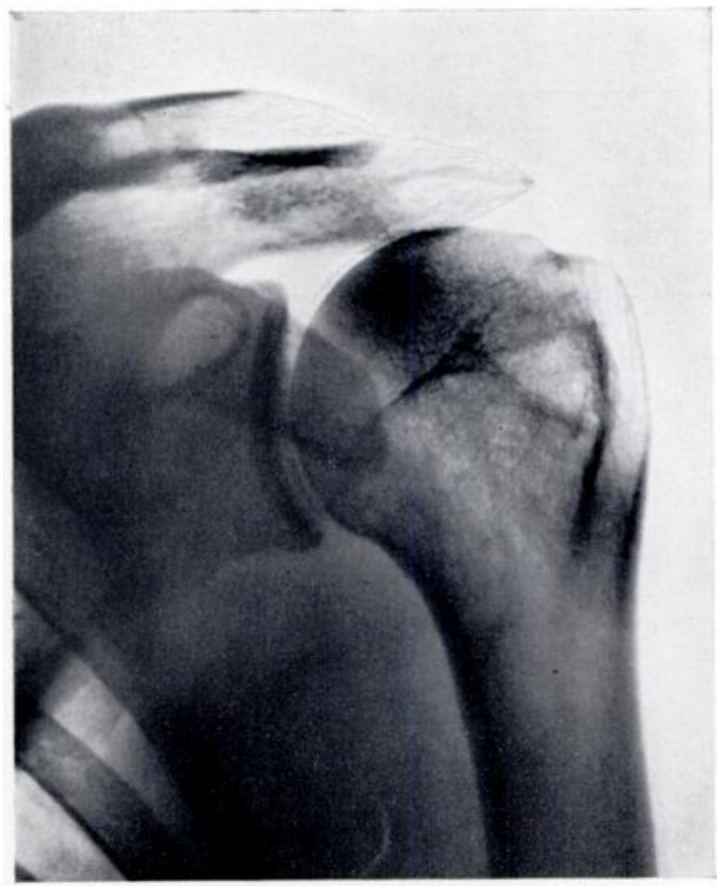

FIG. 3

Left shoulder. Calcified cartilage is seen overlying the head of the humerus and lining the glenoid cavity. up of the cartilage at the edges of the

joint. Both opaque layers were granular, irregular in density, approximately regular in depth and separated from the normal calcified tissue by a thin translucent zone. The general appearance was like that seen in the knees. The right shoulder showed similar but less marked changes. Left carpus -A small triangular opacity was found in the proximal part of the joint between the lunate and the triquetrum, apparently within the articular cartilage of the triquetrum. The other joints of the hand and wrist appeared normal. Spine-Opacities were seen in or immediately deep to the anterior longitudinal ligament at the C.6-7 and T.10-11 intervertebral spaces. The remaining joints and ligaments of the spine were normal. Other cartilaginous tissues-No abnormal calcification was demonstrated in other joints or cartilages. Most of the joints were re-examined five months later but there was no significant change in their appearances.

\section{DISCUSSION}

Calcification near the edge of the intervertebral discs and in the semilunar cartilages of the knees is seen not uncommonly after middle life and might well have been present in this patient independently of the other abnormal calcification. The unusual feature was the calcification of the hyaline articular cartilage. This may be a sign of a generalised disturbance of cartilage metabolism, but such an explanation is unlikely since fibro- and elastic-cartilage elsewhere were not affected. The symptoms were slight, and since there was no indication for opening a joint no histological examination of the tissue has been possible. From the

vol. $35 \mathrm{~B}$, No. 3, August 1953 
radiographs, however, it is evident that there was a deposit of calcium within the superficial layers of the hyaline articular cartilage over the posterior surfaces of the femoral condyles and patellae, over the heads of the humeri and the glenoid fossae, and over the proximal surface of the left triquetrum. At each site the appearance was the same-an irregular flocculent opacity, generally densest towards the surface, with a translucent layer beneath it.

Although the patient had had symptoms in both knees, in the left ankle, and in the right shoulder, there was also abnormal calcification in the left wrist and in the left shoulder, yet both these joints were symptomless. Indeed, the abnormal calcification in the left shoulder was denser and more extensive than in the right. It is possible, therefore, that the symptoms in the right shoulder and both knees could have been due to early osteoarthritic changes alone and not to the calcification in the articular cartilage.

There are several reports in the literature of similar findings. Israelski (1931) published a radiograph of calcification of the semilunar cartilages which also showed a narrow band of calcification lying parallel to the articular surface of the femur, presumed by Israelski to represent an incrustation of calcium on the surface of the articular cartilage. Werwath (1928) described an example of calcification in a semilunar cartilage, detected radiographically. The knee joint was opened; large areas of calcification were seen on the articular cartilage of the femur and tibia and on the medial cartilage, which was removed and sectioned. Small areas of calcification were found in the cartilage. Wolke (1935) examined the radiographs of 2,569 knee joints and found calcification of the cartilages in eight. In five of these patients he described unilateral or bilateral calcification in the articular cartilage of one or more of the following joints: knee, hip, acromio-clavicular, shoulder, elbow, radio-ulnar-carpal, pubic symphysis. He also described calcification in the intervertebral discs. In some instances there was no history of symptoms in the affected joint and, in all patients but one, the symptoms that were present were those of osteoarthritis. Harmon (1944) described two examples of calcification of the articular cartilage over the femoral condyles, and he stated that in one patient similar changes were evident in the wrist joints. The symptoms in one case suggested osteoarthritis; in the other, gout.

We have used the title " primary asymptomatic calcification of articular cartilage" because there is no history of injury to the affected joints and the etiology is obscure; because the condition may clearly be present for long periods before symptoms arise, if they ever do; and because the calcification is within the superficial layers of hyaline articular cartilage, is polyarticular in distribution, but, apart from the constant accompaniment of calcification in the semilunar cartilages, there is no evidence of abnormal calcification in fibro- or elasticcartilage. Whether the calcium is deposited within or around the cartilage cells, or whether it occurs as an incrustation laid down from synovial fluid, can be decided only by histological examination.

\section{REFERENCES}

Harmos, P. H. (1944): Degenerative Calcification in Articular Cartilage of the Knee. Journal of Bone and Joint Surgery, 26, 838.

ISRAELSKI, M. (1931): Meniscus Calcification. Roentgen Findings in Disease of the Menisci. American Journal of Roentgenology, 25, 85 .

Werwath, K. (1928): Abnorme Kalkablagerungen innerhalb des Kniegelenkes, ein Beitrag zur Frage der primären “Meniskopathie." Fortschritte aus dem Gebiete der Röntgenstrahlen, 37, 169.

Wolke, K. (1935): C'ber Meniskus-und Gelenkknorpelverkalkungen. Acta Radiologica, 16, 577. 